\title{
The Monetary Transmission Mechanism in Canada: A Time-Varying Vector Autoregression with Stochastic Volatility
}

\author{
Ronald Henry Lange \\ Correspondence: Ronald Henry Lange, Department of Economics, Laurentian University, Ramsey Lake Road, Sudbury, \\ Ontario, P3E 2C6, Canada.
}

Received: August 26, 2018

doi:10.11114/aef.v5i6.3570

\author{
Accepted: September 27, $2018 \quad$ Available online: October 10, 2018 \\ URL: https://doi.org/10.11114/aef.v5i6.3570
}

\begin{abstract}
This study uses the state-space representation of a time-varying vector autoregression with stochastic volatility (TVP-VAR-SV) to study monetary policy and private sector behaviour in Canada. The main results indicate that both shock variances and autoregressive coefficients of the VAR have evolved systematically over time. The time-varying coefficients of the systematic component of the VAR suggest that monetary policy has become more proactive and less reactive regarding inflation since the early-1990s, which coincides with the adoption of explicit inflation targets. Monetary policy is now able to focus mainly on movements in the output gap to prevent future increases in inflation. The coefficients on the policy rate in both the output gap and inflation equations suggest that the private sector and therefore the transmission mechanism have become more sensitive to monetary policy responses. On the other hand, the coefficients on the output gap in the equations for both inflation and the policy rate have been relatively stable over this period, consistent with view that monetary policy remains more forward-looking regarding inflation than being reactive to inflation surprises as in the past.
\end{abstract}

Keywords: monetary policy, vector autoregession, time-varying parameters, stochastic volatility

JEL Classification: C32, E31, E32, E50

\section{Introduction}

Vector autoregressions (VARs) have now become widely used to study macroeconomic dynamics. Typically, the coefficients in VAR models are assumed to be stable and the error terms are assumed to have constant variances. However, assuming time-invariant coefficients and variances turns out to be quite restrictive in capturing the evolution of most macroeconomic time series. For example, Stock and Watson (2002), and Sims and Zha (2006) have focused on the heteroskedasticity of the exogenous shocks to show that they were particularly volatile in the 1970's and early 1980's. Sims and Zha (2006) also document inferences about monetary policy changes in the U.S. by allowing time variation in both the coefficients of the autoregression equations and variances of the shocks to the macroeconomic variables within a structural VAR framework. The models allowing for changes in the macroeconomic disturbances and the parameters of the monetary policy function were found to be the best-fit models for the US data. The Markov-switching approach has also been employed by Owyang and Ramey (2004) to measure the shifts in the parameters of the monetary policy rule of the Federal Reserve in the U.S.

The regime-switching framework, however, seems to be somewhat limited for analyzing the economy and monetary policy because it assumes a certain number of regimes which have different (but still fixed in time) parameters. The Markov representations in which no state is absorbing permit recurrent shifts, so the system forever switches among the same regimes. The Markov-switching models that assume a finite-state Markov representation permit jumps, but only representations with an absorbing state permit permanent shifts in the variances. Consequently, the framework is somewhat limited by the number of regimes detected in the macroeconomy.

It is now widely accepted that business-cycle dynamics and monetary policy have changed substantially over the post-war period in the U.S., as well as in other countries. Consequently, these changes require allowing time variation in both the coefficients and the variance-covariance matrix in the VAR framework. Some recent research in this area includes Canova and Gambetti (2009), Clark (2009), Cogley and Sargent (2005), Cogley, Primiceri, and Sargent (2010), and Primiceri (2005). These studies find some changes in the transmission mechanism, that is, the way all macroeconomic variables respond to shocks. Particular attention is given to monetary policy because if policy varies 
over time, then this may have a direct effect on the propagation mechanism of the innovations. Furthermore, if agents are rational and forward looking, then the policy changes will be incorporated into the private sector's forecasts of the macroeconomy, inducing some additional modifications in the transmission mechanism. This is the famous Lucas (1976) critique.

The most flexible approach to deal with time variation in the VAR model is where the time variation derives both from the coefficients and variance-covariance matrix of the model's innovations (TVP-VAR-SV). This approach allows both the policy regime and the structure of the economy to be modeled as a gradual evolution of the model's coefficients and heteroskedasticity of the innovations. In the time-varying coefficient approach, parameters are treated as latent variables and assumed to follow a random walk. The Kalman filter is usually applied and maximum likelihood estimation is used for the parameters in a state-space system. Consequently, the estimated parameters for the monetary transmission mechanism are allowed to shift over time in a smooth and gradual fashion. Moreover, by permitting the weights of the policy rule to vary gradually, it is possible to identify relevant shifts in monetary policy conduct, regardless of the policy rule's exact configuration. The time-varying parameter policy-reaction functions may be better suited than conventional fixed parameter or regime-switching equations to deal with the Lucas critique, where parameter changes could change agents' expectations of the future policy course. Also, they may be better suited to handle monetary policy rules where uncertainty about economic conditions is believed to affect the coefficients of the reaction function. In addition, the existence of any type of learning dynamics of private agents or the monetary authorities would favour a model with smooth and continuous drifting coefficients over a model with discrete breaks. In sum, the time-varying VAR approach is preferable because it can capture regime shifts that are smooth transitions rather than abrupt and recurring regime changes as in the regime-switching approach.

The main results of this study indicate that both the shock variances for the economy and the autoregressive coefficients have evolved systematically over time. The relative importance of the non-systematic component for the main macroeconomic shocks was noticeably higher in the first part of the sample during the Great Inflation of the 1970s and early-1980s. The systematic responses of monetary policy indicate a more proactive approach regarding inflation by responding to disturbances in the output gap that signals future inflation than just reacting to inflation surprises as in the past. The time-varying parameters of the systematic component of the VAR also indicate that the private sector responses via the output gap and inflation have become more sensitive to potential monetary policy reactions to these variables.

The paper proceeds as follows. Section 2 briefly outlines some recent research on studies using time-varying parameters for either policy rules or VAR models of the economy. Section 3 presents the state-space representation of the time-varying parameter VAR model with stochastic volatility used in this study for Canada. Section 4 outlines the data and preliminary specification of the model. The empirical results for non-systematic and systematic components of the VAR model are presented and discussed in Section 5. The final section briefly discusses the implication of the results for current monetary policy and discusses some limitations of the study as avenues for future research.

\section{Selective Literature Review}

In the time-varying coefficient framework, many studies have focused just on the monetary-policy reaction function or the Taylor rule. Trecroci and Vassalli (2006), for example, estimated forward-looking Taylor rules for the UK, Germany, France, Italy and the U.S., using the Kalman filter. They demonstrated that the countries analyzed had different interest rate rules and that time-varying Taylor rules are preferred when compared to fixed parameter rules for capturing the variations in the policy rates. The coefficient estimates in their study seldom displayed statistically constant behaviour. Boivin (2006) also estimated a forward-looking Taylor rule using real-time data for the U.S. employing the Kalman filter to construct the likelihood function. Similarly, Kim and Nelson (1990) characterized a forward-looking Taylor rule with time-varying coefficients using ex-post data. Besides the time-varying coefficient property, their study also considered the uncertainty included in the forecasts of future inflation and output gap. Wesche (2003) performed a time-varying coefficient Taylor rule analysis for the countries considered in Trecroci and Vassalli (2006). He reached a similar conclusion using a Markov-switching model with independent switching processes for the time-varying parameters of the Taylor rule and variances of disturbances. Trehan and Wu (2007) employed the Kalman filter for a Taylor rule of the US economy focusing on the time-varying equilibrium real interest rate. They found that taking into account the time-variation in the equilibrium real interest rate made a substantial difference in the assessment of monetary policy. The intuitive appeal of the time-varying parameter approaches in these studies of the monetary policy rule is that the policy regime and structural changes were modeled as a gradual evolution of the model's coefficients.

Some recent studies have used the time-varying parameter VAR approach to analyze the monetary transmission mechanism. Darvas (2009), for example, used the approach to analyze the monetary transmission in three European countries (Czech Republic, Hungary, and Poland) using structural time-varying coefficient vector autoregressions. He 
assumes that the reduced-from VAR parameters follow driftless random walks and uses the state-space representation of the VAR model and the Kalman filter to evaluate the likelihood function. Overall, he found that monetary transmission has changed in all three countries, similar to that of the Euro area. He found that monetary policy has become more effective in Hungary and Poland, but less so in the Czech Republic.

Cogley and Sargent (2005) introduced time varying variances into the context of VARs with drifting coefficients. Their specification forced the variances to adjust continuously to permit recurrent, permanent shifts in the variances. However, in their model the simultaneous relations among variables were treated as time invariant and the standardized VAR innovations were assumed to be independent of the parameter innovations. In general, they found that it is much more difficult to detect evidence for movements in the systematic part of a vector autoregression than to detect stochastic volatility. However, they found enough evidence about drift and stochastic volatility to infer that monetary policy rules have changed and that the persistence of inflation itself has drifted over time.

Following Cogley and Sargent (2005), Doh and Connolly (2012) estimate a time-varying parameter VAR with stochastic volatility with three U.S. macroeconomic variables that include inflation, unemployment and the long-term interest rate. Their analysis suggests that the recession of 2007-2009 was driven by a particularly bad shock to the unemployment rate which increased both its trend and volatility substantially. However, the impacts of the recession on the trend and volatility of nominal variables such as the core inflation rate and the 10-year Treasury bond yield were found to be less noticeable. More recently, Keating and Valcarcel (2017) use annual time series of real output growth and inflation going back well into the 19th century to examine how volatilities of output growth and inflation have varied for different eight countries.

The U.S. study by Primiceri (2005) is particularly relevant for this study of the monetary transmission mechanism in Canada. It overcame one of the main limitations of Cogley and Sargent (2005) by stressing the role of heteroscedastic non-policy innovations. Thus, he allowed for both time variation of the simultaneous relations among the variables of the model and heteroskedasticity of the innovations. This was achieved by developing a simple multivariate stochastic volatility modeling strategy for the variance covariance matrix. He finds that both systematic and non-systematic components of monetary policy have changed during the last 40 year. In particular, he found that systematic responses of monetary policy to inflation and unemployment have exhibited a trend toward a more aggressive behaviour, but that this trend has had a negligible effect on the rest of the economy. Another result is that the exogenous non-policy shocks seem more important than interest rate policy in explaining the high inflation and unemployment episodes in the U.S. In particular, the high volatility of the exogenous non-policy shocks appears to explain a larger fraction of the increases of inflation and unemployment of the 1970's and early 1980's.

Avouyi-Dovi et al. (2017) use the time-varying VAR model with drifting parameters and stochastic volatilities of Cogley and Sargent (2005) and Primiceri (2005) to explore nonlinearities on the French housing and credit markets. They show that variances of the model innovations did not increase during the bubble period of 2000 so that the increased shock variance was not the root cause of the price bubble. However, autoregressive coefficients increased somewhat for housing prices and credit, suggesting some greater shock persistence and stronger price expectations dynamics. The coefficients of the demographic indicator and income also moved upward during the period, indicating an important source of the nonlinearity in their VAR model.

\section{Empirical Methodology}

The econometric framework in this study largely follows Primiceri (2005) to estimate a time varying vector autoregression, where the time variation derives both from both the coefficients and variance-covariance matrix of the model's innovations (TVP-VAR-SV). The state space representation of the VAR is used to estimate the unobserved time-varying coefficients and stochastic volatility in the errors. The time varying parameters are used to measure monetary policy and the multiple equation model of the economy are used to understand how changes in policy have affected the rest of the economy and the monetary transmission mechanism.

Without time-varying coefficients or volatility, the VAR can be estimated by equation-by-equation using ordinary least squares (OLS) which minimizes the sum of residuals in each equation of the VAR. However, by allowing time variation in coefficients and stochastic volatility, the model will include some unobserved components as state variables. To estimate these unobserved components based on the observed data, it is useful to distinguish a state transition equation from the measurement equation as in the canonical representation of a state space model. The estimation of VARs with time-varying coefficients and stochastic volatility in the errors requires the use of filtering methods to extract information about the unobserved states from the observed time series.

The baseline equation of the VAR model is

$$
Y_{t}=\mathrm{c}_{\mathrm{t}}+\mathrm{B}_{1, \mathrm{t}} \mathrm{Y}_{\mathrm{t}-1}+\cdots+\mathrm{B}_{\mathrm{k}, \mathrm{t}} \mathrm{Y}_{\mathrm{k}-\mathrm{t}}+\mu_{\mathrm{t}},
$$


where $Y_{t}$ is an $\mathrm{n} \times 1$ vector of observed endogenous variables; $c_{\mathrm{t}}$ is an $\mathrm{n} \times 1$ vector of time-varying coefficients that multiply or scale up the constant terms; the $B_{i, t} s$ where $i=1, \ldots, k$ are $n \times n$, matrices of time-varying lagged coefficients; and $\mu_{\mathrm{t}} \mathrm{s}$ are the heteroscedastic unobservable shocks with variance-covariance matrix $\Omega_{\mathrm{t}}$. The rectangular reduction of $\Omega_{\mathrm{t}}$ is defined by

$$
A_{t} \Omega_{\mathrm{t}} \dot{A}_{\mathrm{t}}=\sum_{\mathrm{t}} \dot{\Sigma}_{\mathrm{t}}
$$

where $A_{t}$ is a time-varying lower triangle matrix of the time-varying constants $\alpha_{t}$

$$
A_{t}=\left[\begin{array}{cccc}
1 & 0 & \cdots & 0 \\
\alpha_{21, \mathrm{t}} & 1 & \ddots & \vdots \\
\vdots & \ddots & 1 & 0 \\
\alpha_{\mathrm{n} 1, \mathrm{t}} & \cdots & \alpha_{\mathrm{nn}-1, \mathrm{t}} & 1
\end{array}\right] .
$$

The $\alpha_{t}$ s consist of a vector of non-zero and non-one elements of the matrix $A_{t}$ (stacked by rows). Allowing the matrix $A_{t}$ to vary over time is crucial for a time varying VAR where time variation in the simultaneous interactions among variables is fundamental. The matrix $\sum_{\mathrm{t}}$ consist of a vector of diagonal elements in $\sigma_{\mathrm{t}}$ which drive the stochastic volatility processes

$$
\Sigma_{\mathrm{t}}=\left[\begin{array}{cccc}
\sigma_{1, \mathrm{t}} & 0 & \cdots & 0 \\
0 & \sigma_{2, \mathrm{t}} & \ddots & \vdots \\
\vdots & \ddots & \ddots & 0 \\
0 & \cdots & 0 & \sigma_{\mathrm{n}, \mathrm{t}}
\end{array}\right] .
$$

The baseline equation (1) can be written as

$$
Y_{t}=\mathrm{c}_{\mathrm{t}}+\mathrm{B}_{1, \mathrm{t}} \mathrm{Y}_{\mathrm{t}-1}+\cdots+\mathrm{B}_{\mathrm{k}, \mathrm{t}} \mathrm{Y}_{\mathrm{k}-\mathrm{t}}+\mathrm{A}_{\mathrm{t}}^{-1} \sum_{\mathrm{t}} \varepsilon_{\mathrm{t}},
$$

where $\varepsilon_{\mathrm{t}}$ is a standard normal random vector and $\operatorname{VAR}\left(\varepsilon_{\mathrm{t}}\right)=\mathrm{I}_{\mathrm{n}}$ is an $\mathrm{n} \mathrm{x} \mathrm{n}$ identity matrix. Equation (5) can be written in matrix form as

$$
\begin{aligned}
\mathrm{Y}_{\mathrm{t}} & =X_{t}^{\prime} B_{t}+\mathrm{A}_{\mathrm{t}}^{-1} \sum_{\mathrm{t}} \varepsilon_{\mathrm{t}}, \quad \text { and } \\
X_{t}^{\prime} & =\mathrm{I}_{\mathrm{n}} \otimes\left[1, \mathrm{Y}_{\mathrm{t}-1}^{\prime}, \cdots, \mathrm{Y}_{\mathrm{t}-\mathrm{k}}^{\prime}\right],
\end{aligned}
$$

where $X_{t}^{\prime}$ represents the transpose of $X_{t}, \mathrm{~B}_{\mathrm{t}}$ is a vector where the entire right-hand-side time-varying coefficients are stacked, and the symbol $\otimes$ denotes the Kronecker product. Equation (6) is the measurement equation in the state-space representation of the VAR model.

The dynamics of the model's time-varying parameters is specified as

$$
B_{t}=B_{t-1}+v_{t},
$$

where the innovation $v_{t}$ is normal with mean zero and variance-covariance matrix $\mathrm{Q}$. The elements of the vector $\mathrm{B}_{\mathrm{t}}$ are specified as a random walk process, which aims at allowing for the possibility of permanent shifts. In the matrix $A_{t}$ in equation (3)

$$
\alpha_{\mathrm{t}}=\alpha_{\mathrm{t}-1}+\vartheta_{\mathrm{t}},
$$

where the time-varying constants $\alpha_{t}$ evolve according to a drift-less random walk. Following Primiceri (2005), the log volatility terms are log standard deviations instead of $\log$ variances

$$
\log \sigma_{\mathrm{t}}=\log \sigma_{\mathrm{t}-1}+\eta_{\mathrm{t}}
$$

where $\sigma_{\mathrm{t}}$ is the vector of the diagonal elements of $\sum_{\mathrm{t}}$ and the innovation of the volatility $\eta_{\mathrm{t}}$ is normal mean zero with variance $\mathrm{W}$. The standard deviations $\left(\sigma_{\mathrm{t}}\right)$ are assumed to evolve as geometric random walks which belong to the class of models known as stochastic volatility. The model reduces to a VAR with fixed coefficients and stochastic volatility if $v_{t} t=0$ for all $\mathrm{t}$ in equation (7). The equations (7), (8) and (9) are the core relations of the model which explain the evolution of the endogenous variable $\mathrm{Y}_{\mathrm{t}}$ and represent the transition equation for the state-space representation of the model.

The innovations in the model are associated with the following vectors: $\varepsilon_{\mathrm{t}}, v_{t}, \vartheta_{\mathrm{t}}, \eta_{\mathrm{t}}$. They are assumed to be jointly normally distributed with the following assumptions on the variance-covariance matrix 


$$
V=V A R\left(\left[\begin{array}{c}
\varepsilon_{\mathrm{t}} \\
v_{t} \\
\vartheta_{\mathrm{t}} \\
\eta_{\mathrm{t}}
\end{array}\right]\right)=\left[\begin{array}{cccc}
\mathrm{I}_{\mathrm{n}} & 0 & 0 & 0 \\
0 & Q & 0 & 0 \\
0 & 0 & A & 0 \\
0 & 0 & 0 & W
\end{array}\right],
$$

where $I_{n}$ is an $n$-dimensional identity matrix, and $Q, A W$ are positive definite matrices. Each of the four vectors of shocks $\varepsilon_{\mathrm{t}}, v_{t}, \vartheta_{\mathrm{t}}, \eta_{\mathrm{t}}$ contains two elements; $\varepsilon_{\mathrm{t}}$ is a vector of uncorrelated structural shocks with unit variance; Q, A and $\mathrm{W}$ are positive definite $\mathrm{n} \times \mathrm{n}$ matrices; and 0 is a $\mathrm{n}$-dimensional matrix of zeros. None of the off-diagonal zero restrictions are required for estimation. ${ }^{1}$ Following Primiceri (2005) and Cogley and Sargent (2005), the innovations of the model are assumed not to be correlated with each other. The assumption is that $\mathrm{A}$ is block diagonal and the blocks corresponding to the parameters belong to separate equations so that the coefficients of the contemporaneous relations among variables evolve independently in each equation.

Since allowing time variation in coefficients and stochastic volatility introduces too many parameters unless restricted as in Cogley and Sargent (2005), the literature has evolved in a way of introducing random processes to time-varying coefficients and volatility to avoid the "over parameterization" problem. This randomness in these parameters fits quite well with Bayesian methods because there is no strict distinction between fixed "true" parameters and random samples. The goal of Bayesian inference is to obtain joint posterior (post-data) distributions of both the parameters and unobserved states. Since these joint distributions are difficult or impossible to characterize analytically, the time-varying parameter VAR coefficients and stochastic volatility in this study use the Kim, Shephard, and Chib (1998) algorithm to simulate the posterior density using the Markov Chain Monte Carlo (MCMC) method. Gibbs sampling, a particular variant of MCMC method, is used for the posterior numerical evaluation of the parameters of interest to draw from lower dimensional conditional posteriors as opposed to the high dimensional joint posterior of the whole parameter set. The Gibbs sampling iteratively draws parameters and unobserved states conditional on each other to provide draws from the joint distributions under certain regularity conditions to compute the posterior distributions of the parameters of a VAR with time-varying parameters and stochastic volatility.

\section{Data and Preliminary Specification}

The estimation period is from 1963Q1 to 2016Q4 and the frequency is quarterly. Following Primiceri (2005), the first 10 years (40 observations, from 1963QI to 1972Q4) are used to calibrate the coefficients prior distributions. ${ }^{2}$ The macroeconomic variables for the estimations ${ }^{3}$ include and the overnight financing rate in Canada for the monetary policy rate. ${ }^{4}$ The inflation rate is the log-difference over four quarters for the levels of the consumer price index $(2010=100)$ for all items in Canada. The measure is scaled by 100 so that changes can be interpreted as the 4-quarter percentage changes, consistent with the specification of the current inflation targets in Canada. The output gap in Canada is based on GDP (index as 2010=100) and is defined as the log-difference of GDP and trend output scaled by 100. The trend for output in Canada is calculated using a Hodrick-Prescott filter that minimizes the weighted sum of squared deviations from trend and the squared changes in the growth rate of the trend. This weighting is controlled by a smoothness parameter for the ratio of the variances set at 1600 , which is the common choice for quarterly data. ${ }^{5}$ The measure of the output gap builds on a demand/supply separation whereby potential or trend output is the supply side and actual output is the demand side. The real exchange rate is defined as the Canada-US nominal exchange rate (e.g., price of a unit of US currency in terms of Canadian currency) multiplied by the ratio of the US GDP deflator (indexed as $2009=100$ ) to the Canadian GDP deflator (indexed as 2010=100). The real exchange rate is the log-difference over one quarter scaled by 100 so that changes can be interpreted as a 1-quarter percentage change.

\footnotetext{
${ }^{1}$ Primiceri (2005) outlines a minor modification to the estimation scheme to allow for non-zero off-diagonal blocks in equation (10).

${ }^{2}$ The beginning of this sample also coincides with the introduction of flexible exchange rates.

${ }^{3}$ Data for the estimations were retrieved from the OECD database for "Main Economic Indicators" (2010) https://doi.org/10.1787/data-00052-en and available on Federal Reserve Economic Data (FRED).

${ }^{4}$ Armour et al. (1996) find that innovations in the overnight rate, derived using a Choleski decomposition, were consistent with intended policy actions as described in the Bank of Canada's

Annual Reports since the early-1960s.

${ }^{5}$ Hodrick and Prescott motivated their choice of $\lambda=1600$ based on the prior belief that a large change in the cyclical component within a quarter would be around 5 per cent, whereas a large change in the trend component would be around (1/8) per cent, suggesting a choice of $\lambda=\sigma_{c}^{2} / \sigma_{v}^{2}=\left(5^{2}\right) /(1 / 8)^{2}=1600$, where $\sigma_{c}^{2}$ is the variance of the cyclical component and $\sigma_{v}^{2}$ is the variance of the trend component.
} 
The real exchange rate is included as a policy reaction variable because of the Bank of Canada's use of the Monetary Conditions Index (MCI) as an operational target beginning in the late 1980s. ${ }^{6}$ The index is the weighted averages of the monetary policy rate and the exchange rate and it was included as a target variable in monetary policy decisions until the early-2000s. Consequently, monetary policy in Canada at times has responded to disturbances in the foreign exchange market to maintain a stable exchange-rate environment or to achieve a particular level of monetary conditions. Lange (2013), for example, finds evidence of an important role for the real exchange rate in a contemporaneous policy-reaction function for Canada during certain periods. Also, Clarida et al. (1998) suggest lagged values of the exchange rate as possible variables in the central bank's information set at the time it chooses the interest rate.

The data vector for the variables $Y_{t}$ in the vector autoregression is given by

$$
\mathrm{Y}_{\mathrm{t}}=\left(\text { Output }_{\mathrm{t}}, \text { Inflation }_{\mathrm{t}}, \text { Exrate, } \text { Policy } \mathrm{t},\right) \text {, }
$$

where Output $t_{t}$ is the output gap in Canada, Inflation ${ }_{t}$ is the 4-quarter inflation rate for the consumer price index in Canada, and Exrate $_{t}$ is the real exchange rate between the Canadian and U.S. dollars, and Policy ${ }_{t}$ is the overnight financing rate in Canada. The output gap is ordered before inflation because inflation is expected to respond to the changes in the output gap after at least one quarter. Monetary policy is expected to respond to developments in both the output gap, inflation and the real exchange rate in the current quarter.

The choice of lag length for the VAR was guided by the Akaike Information, Schwarz Bayesian and Hannan-Quinn Criteria, which chose 2, 1 and 2 lags, respectively. Consequently, the model was estimated with 2 lags.

\section{Empirical Results}

\subsection{Non-systematic Component of the VAR-Time-Varying Volatilities}

This section assesses whether the variance-covariance of macroeconomic disturbances has changed over time using the stochastic volatility of the errors of the VAR. The simultaneous interaction among the intermediate policy variables are arbitrarily modeled in a lower triangular form in equation (11) so that monetary policy actions affect output or the output gap, inflation and the exchange rate with at least one period of lag. The monetary policy rate is ordered last so that policy can respond to the exchange rate contemporaneously as it did when it was targeting the monetary condition index (MCI). The monetary policy shocks are the measure of non-systematic policy actions that are usually interpreted as capturing "policy mistakes," stochastic shifts in the relative weights given to the intermediate target variables, or policy movements that are responses to variables other than the policy variables and therefore exogenous in this model.

Since the identified shocks are the measures of non-systematic disturbances in the economy, it is natural to measure the relative importance of the changes in non-systematic movements by the time-varying standard deviations of the macroeconomic shocks. Figure 1 presents a plot of the posterior mean, ${ }^{7}$ and the 15 -th and 85 -th percentiles ${ }^{8}$ of the time-varying standard deviation of shocks to the macroeconomic variables, including the monetary policy shock.
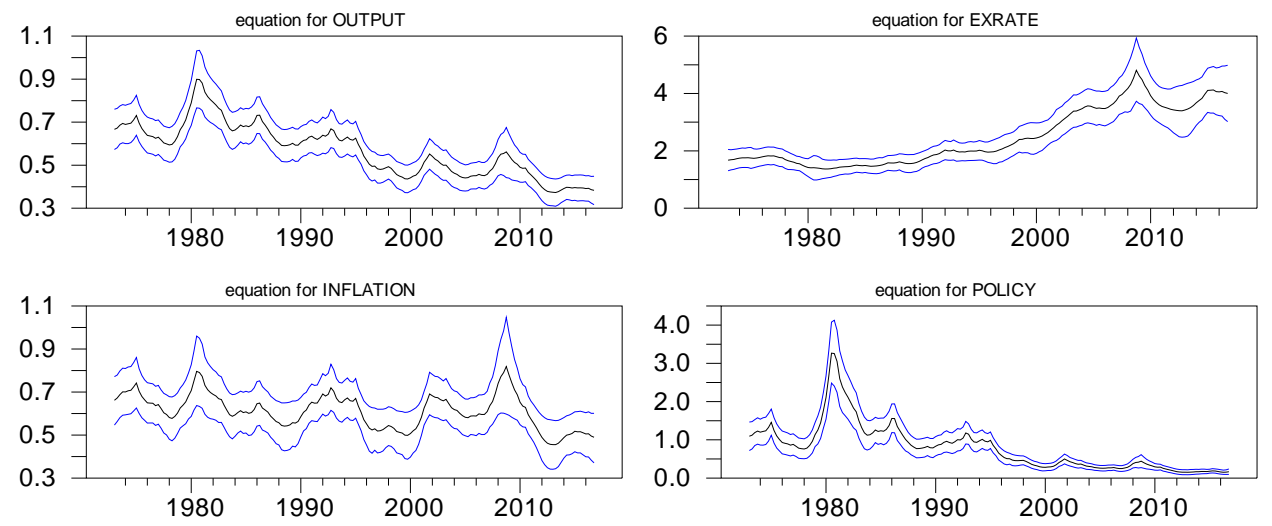

Figure 1. Reduced form posterior means, 15-th and 85-th percentiles for the standard deviations of the residuals of the

\footnotetext{
${ }^{6}$ See Freedman (1994) for a discussion of the use of the MCI by the Bank of Canada.

${ }^{7}$ The posterior density function embodies all the information about the parameters, including the informative priors and the likelihood. The mean of the posterior distribution is the estimator.

${ }^{8}$ Under normality, the 14-th and 86-th percentiles correspond to the bounds of a 1-standard-deviation confidence interval.
} 
equation for the (a) output gap, (b) inflation, (c) exchange rate, and (d) interest rate or monetary policy shocks.

There are two interesting features in the standard deviations in Figure 1. The innovation variances change systematically, being substantially larger in the early-1980s during the Great Inflation for the output gap, inflation and monetary policy than during other times. Also, the innovation variances for both inflation and the exchange rate peaked in late-2009, presumably due to the volatility associated with the Great Recession.

\subsection{Systematic Component of the VAR - Time-Varying Reaction Coefficients}

This section uses the time-varying parameter portion of the VAR to assess whether the propagation of the macroeconomic perturbations has changed over time. The first objective is to capture the responses of the economy to monetary policy shocks to determine whether and how the transmission of monetary policy has changed over time. The second objective is to determine the origin of any change in the transmission mechanism - in particular, whether the change is due to the functioning of the economy or to the change in the conduct of monetary policy itself. Changes in the policy reaction function are likely to affect the way agents form their expectations of future variables and therefore affect the coefficients of the macroeconomic equations in the VAR system. This is the essence of the Lucas (1976) critique.

Figures 2 to 5 present the sums of the two lags of the coefficients for each variable in the equations of the VAR along with the 70 per-cent confidence interval. The equation for the output gap in Figure 2 indicates marginally significant changes in all lagged variables in the early-2000s. More specifically, there are significant small negative coefficients for inflation and the exchange rate and a significant small positive coefficient for the policy interest rate. Figure 3 for the inflation equation indicates that the coefficients for the policy interest rate have become positive and statistically significant since the early-1990s, which corresponds with the introduction of explicit targets for inflation. All parameters except the own lags for the exchange rate in Figure 4 are insignificant. It is noteworthy in Figure 5 for the monetary policy rate that the sums of coefficients on inflation decrease markedly from relatively large positive coefficients to statistically insignificant values in the early-1990s, which coincides with the positive coefficients for the policy rate in the inflation equation in Figure 3.

The most interesting result is the asymmetry in the significance of the lagged coefficients for policy rate in the inflation equation in Figure 3 and the insignificance of the lagged coefficients for inflation in the policy equation in Figure 5. They occur about the same time in the early-1990s when explicit inflation targets were adopted. The insignificance of the lagged coefficients for inflation in the policy equation since early-1990 indicates that monetary policy has not had to respond recently to inflation disturbances like those that were triggered by the supply-side shocks of the 1970s and 1980s. They also suggest that inflation expectations may have become more firmly anchored since the adoption of explicit inflation targets. However, the lagged coefficients on the output gap in the policy equation in Figure 5 have have remained significant and relatively large, although they have declined slightly since the early-1990s. This suggests that that monetary policy has remained proactive and will respond to potential future inflation that may be trigged by shocks to excess demand reflected in the output gap. It is also noteworthy that the coefficients on the lagged policy rate in the policy rate in Figure 5 have increased slightly since the early 1980s. The slight increase suggests that monetary policy has become more inertial and cautious since the 1980s.

Another interesting feature of the time-varying coefficients is the positive and significant coefficients on the policy rate in both the output gap and inflation equations in Figures 2 and 3. This suggests that the private sector and the monetary transmission mechanism have become more sensitive to potential monetary policy responses to increases in the output gap and inflation. 
Equation for OUTPUT
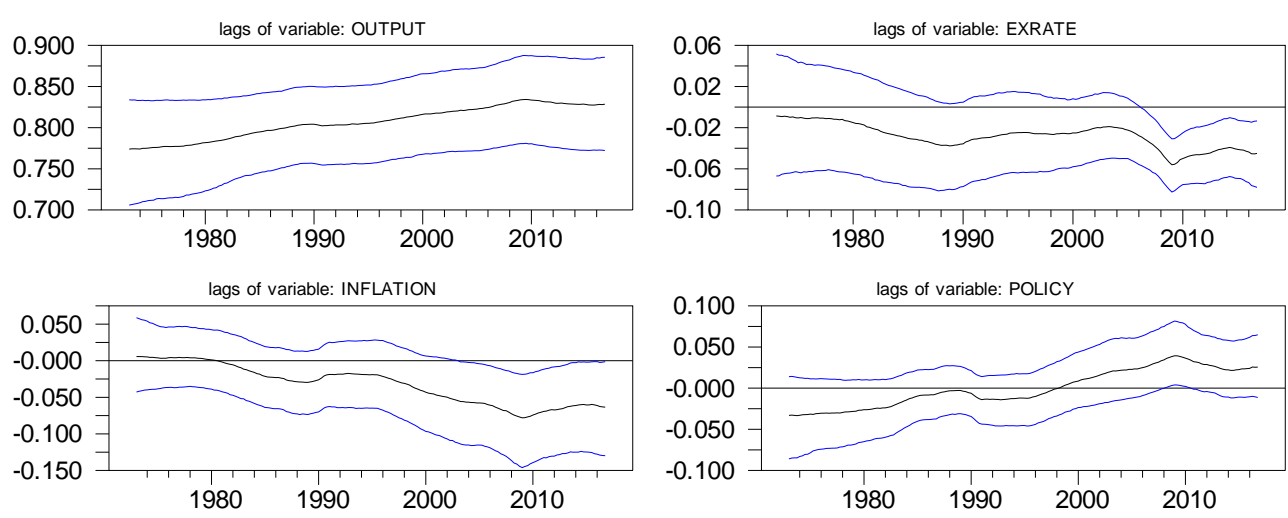

Figure 2. Sums of coefficients for the output equation and 70 per-cent confidence bands.

Equation for INFLATION
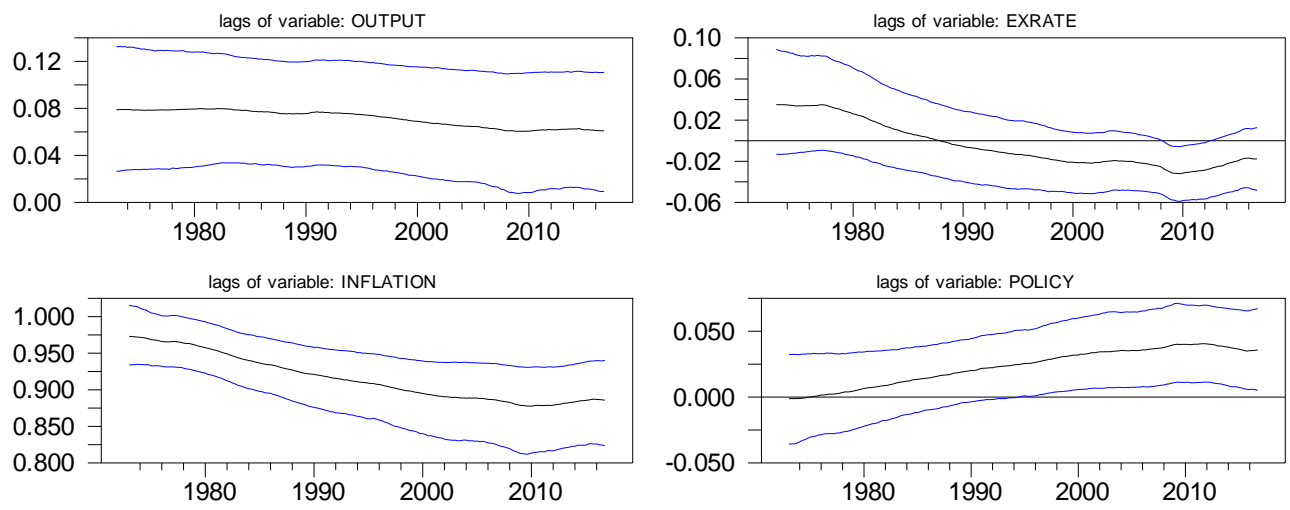

Figure 3. Sums of coefficients for the inflation equation and 70 per-cent confidence bands.
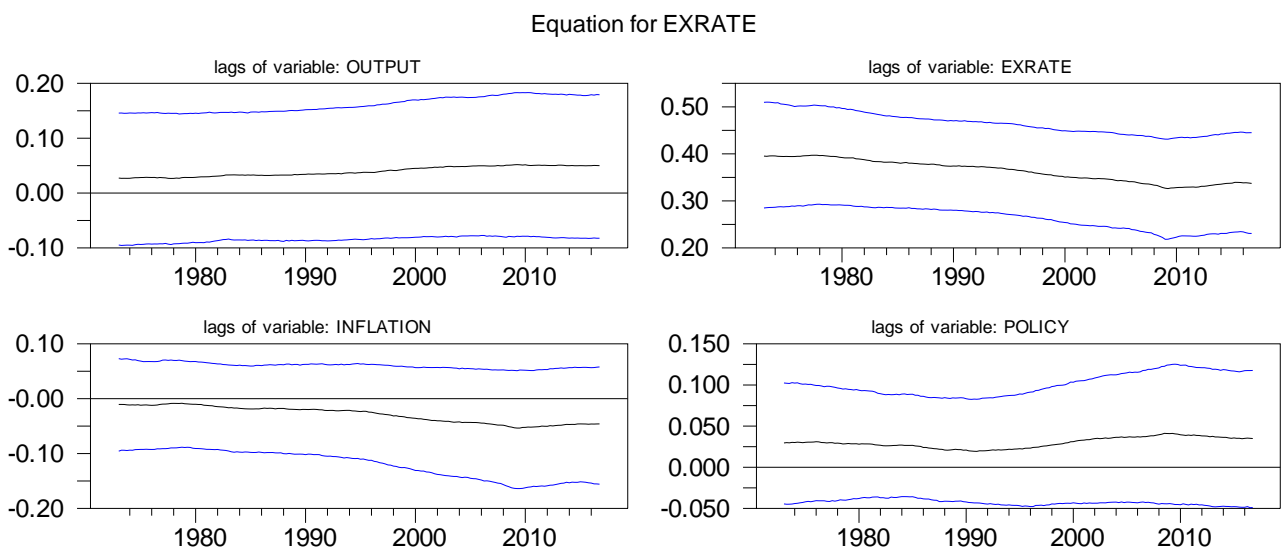

Figure 4. Sums of coefficients for the exchange rate equation and 70 per-cent confidence bands. 


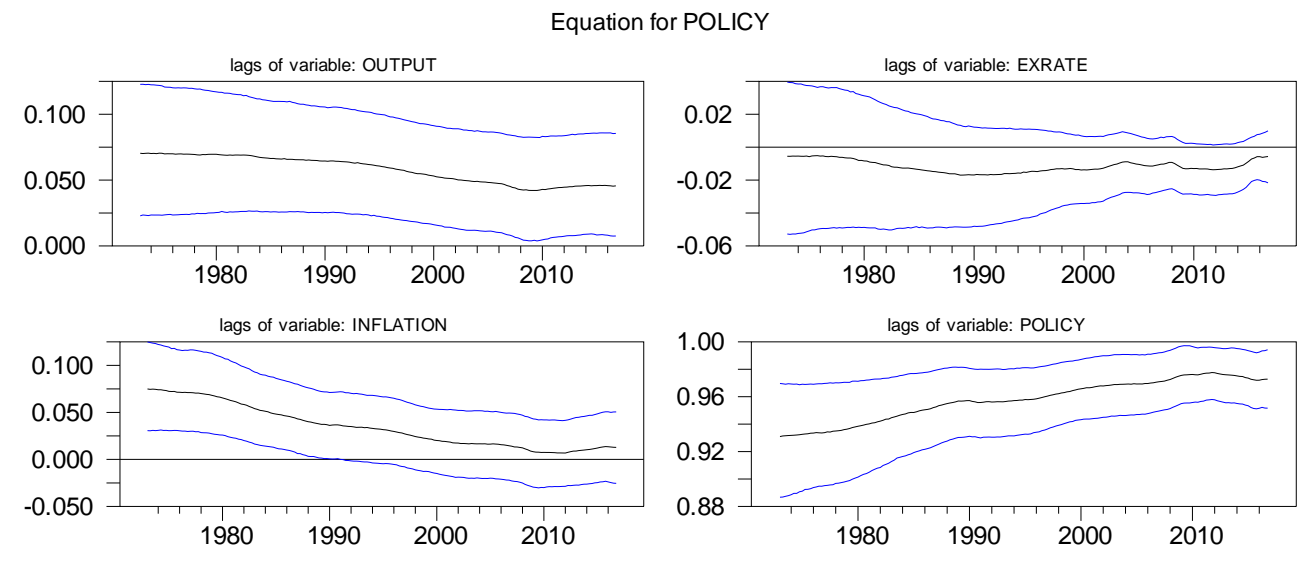

Figure 5. Sums of coefficients for the policy rate equation and 70 per-cent confidence bands.

\section{Concluding Remarks}

The empirical results in this study indicate that both the shock variances and autoregressive coefficients of the TVP-VAR-SV have evolved systematically over time. The relative importance of the non-systematic component of the VAR was significantly higher for the main macroeconomic variables in the first part of the sample during the Great Inflation of the early-1980s. The systematic responses of monetary policy indicate a more proactive approach regarding inflation by responding to disturbances in the output gap that signals future inflation than just reacting to inflation surprises as in the past. The time-varying parameters of the systematic component of the VAR also indicate that the private sector responses via the output gap and inflation have become more sensitive to potential monetary policy reactions to these variables.

The empirical results shed some light on the current monetary transmission mechanism. First, monetary policy has maintained a pre-emptive stance regarding future inflation by remaining focused on the output gap so that it does not have to react to inflation surprises as it had in the past. Second, monetary policy has not focused systematically on the exchange rate as an intermediate information target. Third, the private sector has become more sensitive to potential monetary policy responses to both current inflation surprises and future inflation via the output gap.

One of the main limitations of the current study of the monetary transmission mechanism in Canada is the lack of structure in the VAR model. Further work could allow for a richer set of contemporaneous monetary policy reactions, such as allowing the exchange rate to react concurrently to changes in interest rates. The recent work by Keating and Valcarcel (2017) is also a useful starting point because it allows for the identification scheme of Blanchard and Quah (1989) that decomposes output into permanent and transitory shocks. In addition, a model with an exogenous block could allow for both domestic economic and policy reactions to movements in the U.S. economy. Also, future research could include some financial variables to test whether Canadian monetary authorities have targeted other intermediate variables, such as the yield curve or the term spread.

\section{References}

Armour, J., Engert, W., \& Fung, B. (1996). Overnight innovations as a measure of monetary policy shocks in vector autoregressions. Bank of Canada Working Paper, No. 96-4.

Avouyi-Dovi, S., Labonne, C., Lecat, R., \& Simon, R. (2017). Insight from a time varying VAR model wit stochastic volatility of the French housing and credit markets. Bank of France Working Paper, No. 620.

Blanchard, O., \& Quah, D. (1989). The dynamic effects of aggregate demand and supply disturbances. The American Economic Review, 79, 655-673. https://doi.org/10.3386/w2737

Boivin, J. (2006). Has U.S. monetary policy changed? Evidence from drifting coefficients and real-time data. Journal of Money, Credit and Banking, 38(5), 1149-1173. https://doi.org/10.1353/mcb.2006.0065

Canova, F., \& Gambetti, L. (2009). Structural changes in the US economy: Is there a role for monetary policy? Journal of Economic Dynamics and Control, 33, 477-490. https://doi.org/10.1016/j.jedc.2008.05.010

Clark, T. (2009). Is the Great moderation over? An empirical analysis, Economic Review, Federal Reserve Bank of Kansas City, 5-42.

Cogley, T., \& Sargent. T. (2005). Drifts and volatilities: Monetary policies and outcomes in the Post WWII 
U.S.,"Review of Economic Dynamic, 8(2), 262-302. https://doi.org/10.1016/j.red.2004.10.009

Cogley, T., Primiceri, G., \& Sargent, T. (2010). InfIation-gap persistence in the US. American Economic Journal: Macroeconomics, 2(1), 43-69. http://dx. doi.org/10.1257/mac.2.1.43

Darvas, Z. (2009). Monetary transmission in three central European economies: Evidence from time-varying coefficient vector autoregressions. De Nederlandsche Bank Working Paper, No. 208.

Doh, T., \& Connolly, M. (2012). The state space representation and estimation of a time-varying parameter VAR with stochastic volatility. Federal Reserve Bank of Kansas City, Research Working Paper, 12-04.

Freedman, C. (1996). The use of indicators and of the monetary conditions index in Canada. In The Transmission of Monetary Policy in Canada, 67-80, Bank of Canada.

Gelfand, A., \& Smith, A. (1990). Sampling-based approaches to calculating marginal densities. The Journal of the American Statistical Association, 85, 398-409.

Keating, J. W., \& Valcarcel, V. J. (2017). What's so great about the Great Moderation? Journal of Macroeconomics, 51, 115-142. https://doi.org/10.1016/j.jmacro.2016.11.006

Kim, C. J., \& Nelson, C. R. (2006). Estimation of a forward-looking monetary policy rule: A time-varying parameter model using ex-post data. Journal of Monetary Economics, 53(8), 1949-1966. http://dx.doi.org/10.1016/j.jmoneco.2005.10.017

Kim, S., Shephard, N., \& Chib, S. (1998). Stochastic Volatility: Likelihood Inference and Comparison with ARCH Models. Review of Economic Studies, 65, 361-393. https://doi.org/10.1111/1467-937X.00050

Lange, R. (2013). Monetary policy reactions and the exchange rate: a regime-switching structural VAR for Canada. International Review of Applied Economics, 27, 612-632. https://doi.org/10.1080/02692171.2012.752446

Lucas, R. E., Jr. (1976). Econometric policy evaluation: A critique. Carnegie-Rochester Conference Series on Public Policy, 1, 19-46.

Owyang, M., \& Ramey, G. (2004). Regime switching and monetary policy measurement. Journal of Monetary Economics, 51, 1577-1597. https://doi.org/10.1016/j.jmoneco.2004.01.004

Primiceri, G. (2005). Time-varying structural vector autoregressions and monetary policy. Review of Economic Studies, 72, 821-852. https://doi.org/0034-6527/05/00330821\$02.00

Sims, C., \& Zha, T. (2006). Were there regime switches in macroeconomic policy? American Economic Review, 96(1), 54-81. http:// dx.doi.org/ 10.1257/000282806776157678

Stock, J. H., \& Watson, M. W. (2002). Has the Business Cycle Changed and Why? National Bureau of Economic Research, Working Paper, No. 9127.

Trecroci, C., \& Vassalli, M. (2006). Monetary policy regime shifts: New evidence from time- varying interest rate rules. University of Brescia, Department of Economics, Discussion Paper 0602.

Trehan, B., \&Wu, T. (2007). Time-varying equilibrium real rates and monetary policy analysis. Journal of Economic Dynamics and Control, 31(5), 1584-1609. https://doi.org/10.1016/j.jedc.2006.04.009

Wesche, K. (2006). Estimating central banks' preferences from a time varying empirical reaction function. European Economic Review, 50, 1951-1974 https://doi.org/10.1016/j.euroecorev.2005.10.003

\section{Copyrights}

Copyright for this article is retained by the author(s), with first publication rights granted to the journal.

This is an open-access article distributed under the terms and conditions of the Creative Commons Attribution license which permits unrestricted use, distribution, and reproduction in any medium, provided the original work is properly cited. 